\title{
MESOPOROUS SILICA FUNCTIONALIZED BY NICKEL-CYCLAM MOLECULES: PREPARATION AND RESONANCE RAMAN STUDY
}

\author{
MAGDALENA LASKOWSKA ${ }^{1}$, LUKASZ LASKOWSKI $^{2}$, KAZIMIERZ DZILINSKI $^{1}$
}

1. Institute of Physics, Czestochowa University of Technology, PL-42-200 Czestochowa, Al. Armii Krajowej 19, Poland. 2. Department of Computer Engineering, Czestochowa University of Technology, PL-42-200 Czestochowa, Al. Armii Krajowej 36, Poland.

Received February 13, 2012; accepted May 7, 2012; published online May 14, 2012

\begin{abstract}
Mesoporous silica SBA-15 functionalized by (1,4,8,11-tetraazacyclotetradecane) cyclam groups containing nickel ions (Ni-cyclam) was synthesized by two different approaches, and investigated by resonance Raman spectroscopy. Vibrational features of organometallic moleculess are analyzed for (Ni-cyclam) groups grafted in the silica pores. An assignment of bands in resonance Raman spectra was done to monitor the structure and properties of the mesoporous silica material with regard to the methods of synthesis used in this study. It was shown, that Raman scattering can be useful for probing of functionalization's efficiency of mesoporous silica. On the base of the resonance investigation: Raman and EPR spectroscopy, distribution of the functional groups inside pores can be determined. In the present article the Raman spectroscopy is treat as a complementary research to EPR investigation.

It was shown that a clustering of the active groups alter significantly the resonance Raman spectra through broadening and shifts of the corresponding bands in comparison with separated molecules. Results obtained from the analysis of the resonance Raman spectra indicate significant differences between the samples prepared by the two procedures. The discussion of the Raman results was referred to EPR results, and on the base of this authors concluded about correct achievement of the functionalization.
\end{abstract}

\section{INTRODUCTION}

Nanotechnology represents certainly one of the most promising developments of industry. One of the major challenges in this field is the control of physical or chemical properties of materials through modeling their structure in the nanometric scale. Elaboration of the synthesis route of MCM-41 mesoporous silica (Beck, Vartuli, Roth, Leonowicz, Kresge, Schmitt, Chu, Olson, Sheppard, McCullen, Higgins, Schlenkert, 1992) was a milestone in nanotechnology. This inorganic material has an open structure and a well-defined geometry. Mesoporous silica has a form of thin and long rods with walls built with amorphous silica $\left(\mathrm{SiO}_{2}\right)$. The rods arrangement is usually regular, often hexagonal. Development of composite materials, such as mesoporous silica, containing pores which size can be easily and accurately controlled, has a great importance for science and technology. Nowadays, it is possible to obtain mesoporous silica with pores in the hexagonal, cubic or lamellar arrangement with diameter from 1,5 to $15 \mathrm{~nm}$ depending on synthesis conditions and a type of a surfactant.

Mesoporous silica SBA-15 (Santa Barbara Amorphous) (Zhao, Huo, Feng, Chmelka, Stuc, 1998) is the main object of investigation in this paper. The structure of this material is shown in Fig.1. a)

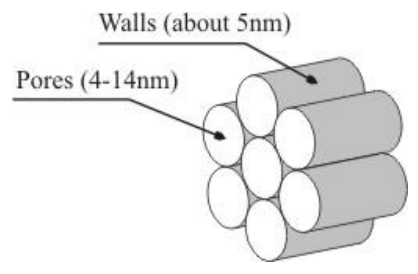

b)

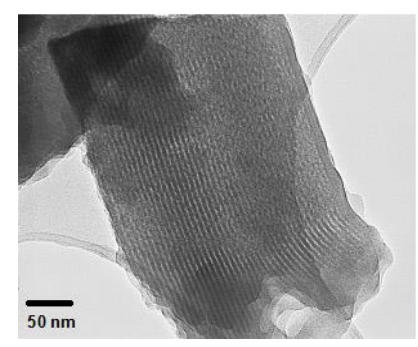

Fig.1. The structure of SBA-15 type mesoporous silica: scheme (a) and microphotography (b) 
The SBA-15 was worked out at the University of California in 1996. This unusually stable material is non-toxic as well as relatively cheap and easy to produce.

In the pure form SBA-15 has a very limited application. The potential applications are connected with the functionalization by different chemical compounds (Corriu, Mehdi, Reye, 2004).

a)

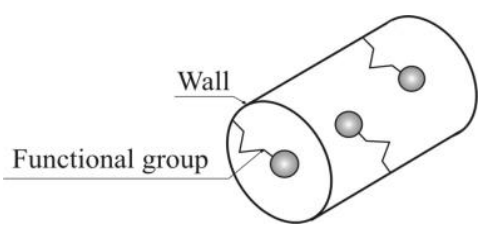

Synthesis cannot be a purpose in its own right but it should be designed precisely to yield a material showing properties expected for the specific application. Some molecules can be attached to inside pores and walls giving structures which show specific properties for some applications (Corriu, 2003). The functional groups localized inside pores and between them are shown in Fig. 2.

b)

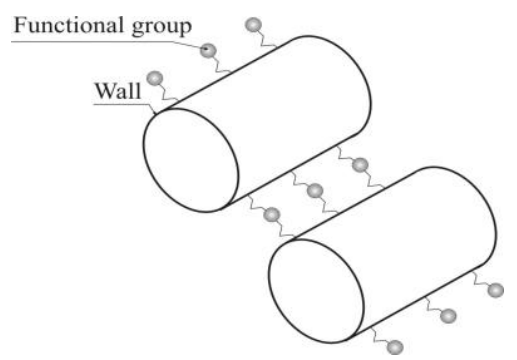

Fig. 2. The way of mesoporous silica functionalization: inside pores (a) and in wall structures (b).

Many synthesis procedures have been oriented to mesoporous silica with variable pore sizes $(2-20 \mathrm{~nm})$. It should be noticed, that there are possibilities to control precisely localization of the functional groups, both inside pores and in walls (Corriu et al., 2004; Corriu, 2003). The functionalization procedure can be carried out by grafting or by direct synthesis. Both routes lead to materials with different properties (Laskowski, Kassiba, Makowska-Janusik, Mehdi, Gibaud, Errien, Swiatek, 2009). The main challenge is connected with production of samples containing functional groups homogeneously distributed in the host matrix. One of essential requirements in this field is fine tuning of the physical properties (electronic, magnetic, optical) to the hybrid architectures depending on the nature of active groups.

SBA-15 mesoporous silica has been applied in medicine and as elements of biosensors. For the sake of great volume of pores (reaching $1000 \mathrm{~m}^{2} / \mathrm{g}$ ) it is possible to accumulate the medicines inside them. The mesoporous silica plays in medicine a role of the Trojan horse (Katiyar, Yadav, Smirniotis, Pinto, 2006; Trewyn, Nieweg, Zhao, Lin, 2007). The particles of silica containing a medicament, can penetrate inside the cells (by endocytosis) and next release the drug in them. This feature offers new possibilities in some kind of cancer treatment. Besides, SBA-15 can be used to improve availability of the hydrophobic drugs (e.g. Itraconazol) in such a way that after the placement of a medicament in the silica pores, mesoporous silica is next absorbed in a stomach and intestines.

As far as biosensors are concerned, the SBA-15 has a great possibility of application (Slowing, Trewyn, Giri,
Lin, 2007). Thanks to variety of molecules, attaching to inside pores and inside walls, functionalized particles of mesoporous silica can be compatible with particular types of cells. The mesoporous silica containing fluorescent dye, after infiltration into target cells, can be used to mark these cells.

A great volume of pores in mesoporous silica creates a possibility of application in separation and recycling (Jung, Hartmann, 2008). The mesoporous silica functionalized by adequate molecules, can capture particular molecules into channels (inside pores) which should be recovered. Silica creates suspensions with many solvents (this material is not soluble even in strong acids), while recovered material can be easily solved and next obtained from suspension by filtering. The great advantage of the SBA-15 is its good stability, also that one functionalized by cyclam, which shows strongly chelating properties.

The mesoporous silica is used also for catalysis (Xiao, 2005). Porous nanopowder plays in this case a role of a specific catalytic agent. The total content of active ions depends, inter alia, on a surface of support and the active surface, in the case of SBA-15, is very large. Therefore catalytic systems based on mesoporous matrices are characterized by unusually high catalytic activity.

The most attractive, from the financial point of view, is the application of mesoporous silica materials in optoelectronics, as optical modulators in data transferring systems (Lebeau, Sanchez, 1999). Silicabased optoelectronic chips are much cheaper than systems built on the base of semiconductors. The light is split into two beams in an optical modulator and next the beams are overlapped at the chip output. If one beam is 
shifted in phase in relation to the other, the beams are quenched and this situation corresponds to zero bit. Otherwise, a bit has value equal to one. The phase shift of light beams in optical modulator can be caused by the difference in charge carriers concentration. Thanks to a possibility of the grafting of a low-symmetry dipolar compound into SBA-15 mesoporous silica it is possible to obtain optically an active material (dipolar moments of functional groups interact with light beam), which is able to polarize light beam crossing the material (by the way, this property can be useful for photography).

This paper deals with mesoporous silica functionalized by (1,4,8,11-tetraazacyclotetradecane) chelating nickel ions (Ni-cyclam). It contains a description of synthesis and an analysis of the active bands in the resonance Raman spectra. Different samples were investigated including pure (Ni-Cyclam) powders, pure mesoporous silica and the functionalized ones. The Raman experiments were carried out in the same conditions for matrices functionalized by different ways.

\section{EXPERIMENTAL}

\section{Synthesis}

All reactions were carried out under argon atmosphere by using a vacuum line. Solvents were dried and distilled just before the use. Triblock copolymer $\left(\mathrm{EO}_{20} \mathrm{PO}_{70} \mathrm{EO}_{20}\right)$ with $\mathrm{EO}$ (poly-ethylene oxide) and $\mathrm{PO}$ (poly-propylene oxide), Pluronic P123, CPTES (3- chloropropyltriethoxysilane)

and

TEOS (tetraethylorthosilicate) were purchased from Aldrich and used as supplied. The molecules (1,4,8,11tetraazacyclotetradecane), hereafter called cyclam, were purchased from Chematech and $\mathrm{NiCl}_{2}$ from Acros. The specific surface areas of the different samples were determined by Brunauer-Emmett-Teller (BET) method.

Two families of the mesoporous materials functionalized by Ni-Cyclam groups were synthetized. They are referenced below as (SA5, SA10) and (SB5, SB10) with regard to the loading rates of Ni-cyclam about $5 \%$ and $10 \%$. Both families of the samples have originally the same structure, but they differ in the preparation procedure of the pre-functionalized mesoporous silica based on SBA-15 backbones and grafted chlorine ions (Cl-SBA). The main difference in the synthesis routes is following: the SA samples were obtained by grafting of chloropropyl and next the cyclams into mesoporous silica matrix whereas the SB samples containing chlorine in SBA-15, were obtained by the one-step synthesis.

To define correctly the doping rates, we refer to the molar ratios between (3-chloropropyltrimethoxysilane) as a precursor for cyclam grafting and the silica units from TEOS. A doping rate of $10 \%$ means the use of 1 $\mathrm{Cl}-\mathrm{Si}(\mathrm{OEt})_{3}$ for 9 TEOS units (samples with index of 10). Similarly doping rate of $5 \%$ means the use of $1 \mathrm{Cl}$ $\mathrm{Si}(\mathrm{OEt})_{3}$ for 19 TEOS units (samples with index of 5). The main differences between series of the samples and the synthesis procedure can be seen in fig. 3 .

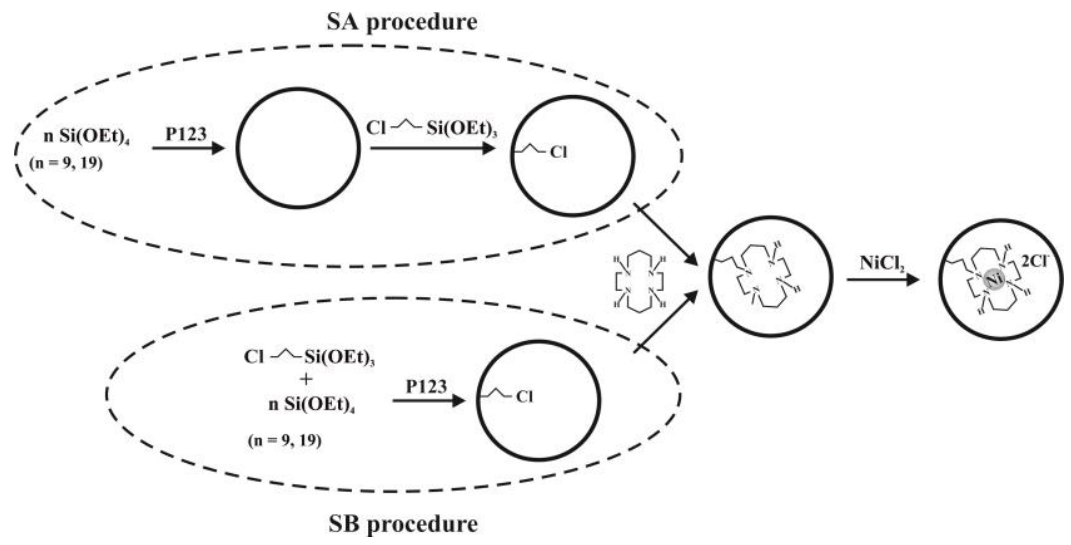

Fig. 3. Schematic representations the synthesis procedures to functionalize the mesoporous silica pores by nickel - cyclam groups. SA samples are obtained by two steps of synthesis according to the described SA procedure. SB samples are obtained by one step synthesis process as illustrated by SB procedure.

The preparation of a samples is described below.

SA samples: pre-functionlized [CI-SBA]. The surfactant - Pluronic P123 was dissolved in comparatively high acidic aqueous solution of $\mathrm{HCl}(2 \mathrm{M})$. After about $1 \mathrm{~h}$ stirring the clear solution was obtained. The measured amount of TEOS was added to the resulting solution and vigorously stirred for 45 minutes at temperature of $40^{\circ} \mathrm{C}$ in hot oil bath in order to start polycondensation of silane groups. After this time a white suspension appeared indicating that a mesoporous material was formed.

The second step deals with the grafting of the active groups through the CPTES added to a suspension which contains hexagonal arranged mesoporous silica. The 
amount of the CPTES depends on the doping rate, we wont to obtain. In order to initialize grafting process, tightly closed flask with liquid was put into oven $\left(90^{\circ} \mathrm{C}\right)$ for one night. The resulting white powder was filtered off and the surfactant was selectively removed by soxhlet extraction over ethanol for 24 hours. After drying at $120^{\circ} \mathrm{C}$ overnight under vacuum, Cl-SBA were obtained as white powder.

\section{SB samples: pre-functionlized [Cl-SBA]}

This procedure is an direct synthessis as opossed to the procedure described before. According to scheme (see: fig. 1.) pluronic P123 was dissolved in aqueous solution of $\mathrm{HCl}(\mathrm{pH}=1.5)$. The resulting clear solution was then added to a mixture of 3chloropropyltriethoxysilane and TEOS (mixed in appriopriate proportions, depending on the desirable doping rate). The mixture was stirred for 3 hours at room temperature until a transparent solution appeared, what means the homogenous mixture was obtain. The solution was transferred into a hot oil bath at $60^{\circ} \mathrm{C}$. NaF (catalyser) was then immediately added to induce the polycondensation. A white precipitate appeared within a few minutes and the resulting suspension was further stirred for 2 days at $60^{\circ} \mathrm{C}$. The resulting white powder was filtered off and the surfactant was selectively removed by soxhlet extraction over ethanol for 24 hours. After drying at $120^{\circ} \mathrm{C}$ overnight under vacuum , the $\mathrm{Cl}$ SBA were obtained as a white powder. Specific surface measurements give $890 \mathrm{~m}^{2} \mathrm{~g}^{-1}$ and the pore volume 1.1 $\mathrm{cm}^{3} \mathrm{~g}^{-1}$.

a)

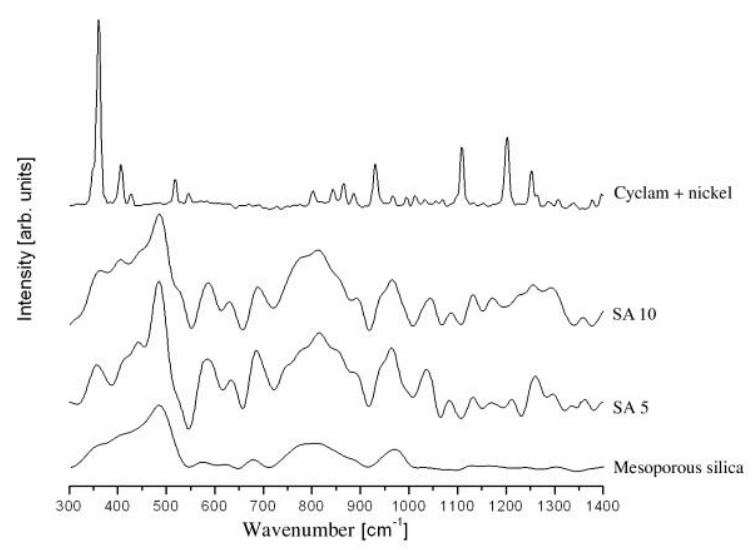

Reactivity of chloropropyl groups towards cyclam: [cycl-SBA]

The preparation of cycl-SBA uses a mixture of cyclam and $\mathrm{Cl}-\mathrm{SBA}$ dissolved in $\mathrm{f}$ acetonitrile and triethylamine. The product was then heated under reflux and stirring for 2 days. The white solid was quantatitatively recovered by filtration and washed 5 times with hot chloroform and 3 times with hot ethanol to remove the excess of cyclam. After drying at $120^{\circ} \mathrm{C}$ overnight under vacuum, cycl-SBA were obtained as a white powder.

\section{Complexation by nickel salts: $\mathrm{Ni}$-cycl-SBA}

A small amount of cycl-SBA powder was added to ethanol solution of $\mathrm{NiCl}_{2}$. The proportion between species depends on the doping rate. The resulting suspension was heated overnight under reflux with stirring. The solid was quantitatively recovered by filtration and washed with dry ethanol several times to remove the excess of nickel salt. After drying at $120^{\circ} \mathrm{C}$ overnight under vacuum, $\mathrm{Ni}$-cycl-SBA was obtained as green powder.

Raman spectroscopy. A confocal Raman spectrometer Jobin-Yvon-Horiba T64000 was used at room temperature. The experiments were done by selecting the $514 \mathrm{~nm}$ ( $\mathrm{Ar}^{+}$laser) or the $647 \mathrm{~nm}\left(\mathrm{Kr}^{+}\right.$laser) radiations with the power about $40 \mathrm{~mW}$. The experimental conditions were the same for all the investigated samples. Due to the lack of the resonance Raman spectroscopy data in the literature concerning the cyclam based mesoporous matrices, the spectra were registered in a wide range of the wave numbers.

b)

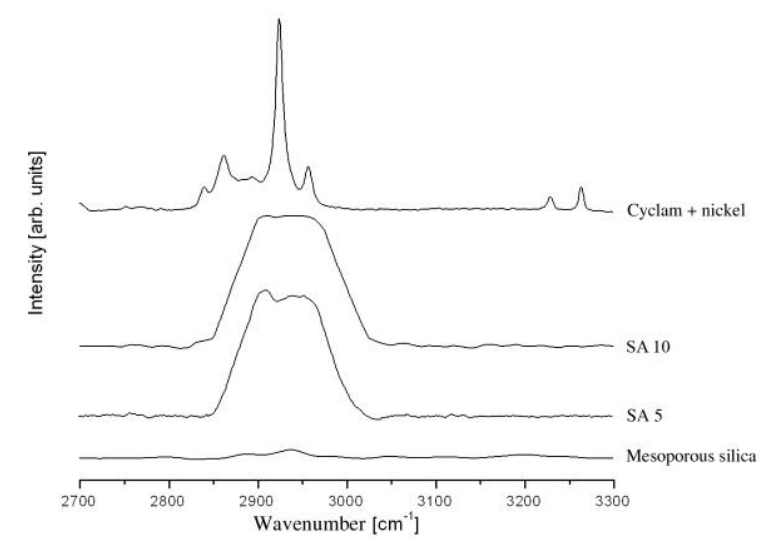

Fig. 4. Low (a) and high wavenumber (b) range of resonance Raman spectra obtained from mesoporous silica with cyclam groups inside channels (SA11). The Raman spectra for the cyclam groups alone and pure silica are also reported for comparison. 


\section{RESULTS AND DISCUSSION}

\section{Resonance Raman spectra}

In the case of the SA samples, similar features of the resonance Raman spectra are observed irrespective of the quantity of the doped compound (Fig. 4).

In contrast to the SA samples, the SB samples show the resonance Raman spectra containing bands which

a)

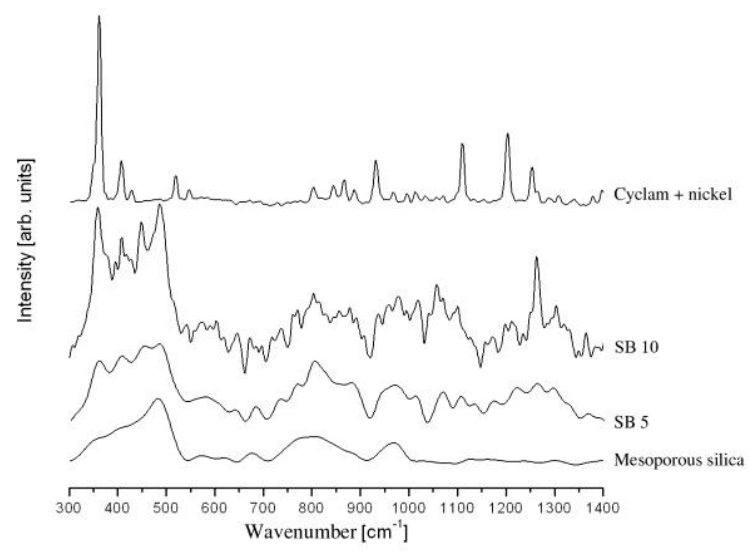

correlate with the quantity of the active groups (Fig. 5). The Raman bands of functionalized silica consist of contributions from the active molecules and the host matrix. However, the experiments show very weak Raman bands from silica, while pure Ni-cyclam molecules give a rise to well-resolved resonance Raman signals. An analysis of the Raman bands is discussed below.

b)

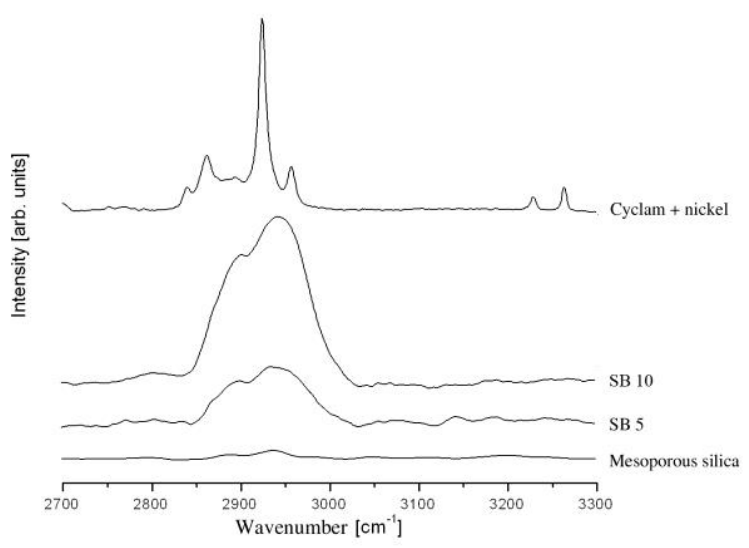

Fig. 5. Low (a) and high (b) frequency part of the resonance Raman spectra of mesoporous silica with active cyclam groups inside channels (SB5 and SB11 samples).

Pure Ni-Cyclam groups. The weak bands at $2837 \mathrm{~cm}$ 1 and $2860 \mathrm{~cm}^{-1}$ result from symmetric $\mathrm{CH}_{2}$ stretching (Singh, Singh, Amareshwar, Rai, Singh, Rai, 2007) while the large-intensity one at $2923 \mathrm{~cm}^{-1}$ can be assigned to asymmetric stretching of $\mathrm{CH}_{2}$ groups (Otakar, Jehlicka, Howell, 2007). The shift at $2959 \mathrm{~cm}^{-1}$ is related to $\mathrm{CH}$ group vibrations ( $\mathrm{Xu}$, Butler, Gibson, Stangel, 1997). Next two week shifts can be observed at 3230 and $3260 \mathrm{~cm}-1$. The origin of this features is stretching vibrations of $\mathrm{N}-\mathrm{H}$ groups. Characteristic frequency for this vibration mode is 3250 , however lack of the equivalence in local environment of this group can cause splitting of the vibrational band into two components, what can be observed in this case (Smierciak, Passariello, Blinn, 1977).

In the low wave-number range, the resonance Raman shifts at $890 \mathrm{~cm}^{-1}$ are assigned to $\mathrm{CH}_{2}-\mathrm{N}-\mathrm{CH}_{2}$ deformation while the band at $1107 \mathrm{~cm}^{-1}$ can be associated with $\mathrm{N}-\mathrm{CH}_{2}$ longitudinal vibrations (Hess, Wild, Schlögl, 2006). However, the main characteristic band near $370 \mathrm{~cm}^{-1}$ seems to be related to the nickelamine bonds which form Ni-cyclam groups (Hess, 2006; Davidson, 2005). This large intensity band can be considered as a criterion for the identification of the nickel-cyclam complexes in the mesoporous silica.

Pure mesoporous silica. In the case of pure silica, we have used known reports [Liao, Li, Hao, Ai, Zhang, Wang (2007); Jones, Goldcamp, Krause, Baldwin
(2006)] to assign the origin of the resonance Raman peaks. The broad resonance Raman band around 480 $\mathrm{cm}^{-1}$ can be assigned to D1 defect mode of cyclic tetrasiloxane rings of the silica support. The Raman shift around $980 \mathrm{~cm}^{-1}$ is typical of the $\mathrm{Si}-\mathrm{OH}$ stretching vibration modes which occurs from hydroxyl groups. The weak Raman band at $810 \mathrm{~cm}^{-1}$ is induced by the symmetrical $\mathrm{Si}-\mathrm{O}-\mathrm{Si}$ stretching mode.

Functionalized mesoporous silica. The effect of the Ni-cyclam in the silica matrix on the resonance Raman spectra is much more noticeable in the case of the SB5 and SB10 samples than the SA5 and SA10 ones. In the wavenumber range $2850 \mathrm{~cm}^{-1}-3050 \mathrm{~cm}^{-1}$, the resonance Raman spectra of the SA samples exhibit a structureless broad band. Its intensity increases slightly with the quantity of the doped compound and correlates mainly with the $\left(\mathrm{CH}_{2}\right)_{\mathrm{i}}$ chains of the functional groups localized in the mesoporous silica channels. The vibrational bands from the cyclam groups are no longer resolved. In the case of SB samples strong dependence of Raman features on the doping rate can be observed at this range. The peeks takes from vibrations of $\mathrm{CH}$ and $\mathrm{CH}_{2}$ groups in cyclam molecules are much more resolved in the case of this samples batch, on the contrary to SA ones.

In the low wave-number range, the resonance Raman spectra of the SA samples are mainly marked by the weak silica Raman bands without a clear evidence of 
contributions from the cyclam groups. This feature is not observed in the resonance Raman spectra from the SB samples where the relevant bands from the cyclam groups are resolved. Particularly, the intensity of the Raman band at $370 \mathrm{~cm}^{-1}$, from the nickel complexed cyclam, correlates with the quantity of the doped species (Fig. 5a). The unresolved bands in the remaining part of the resonance Raman spectra (high wave-number range) are caused by the same reasons as in the case of the SA samples. These broad unresolved bands result probably from interactions between the mesoporous matrix backbone and the functional groups or from agglomeration effects. Both causes can lead to a distribution of vibration modes and next to broadening of the resonance Raman bands.

\section{Reference to EPR investigation}

The continuous wave electron paramagnetic resonance spectroscopy (CW-EPR) was used to characterization of the effectiveness of the samples synthesis and its functionalization by the nickel-cyclam groups. Detailed discussion of this results was presented in (Laskowski et

a)

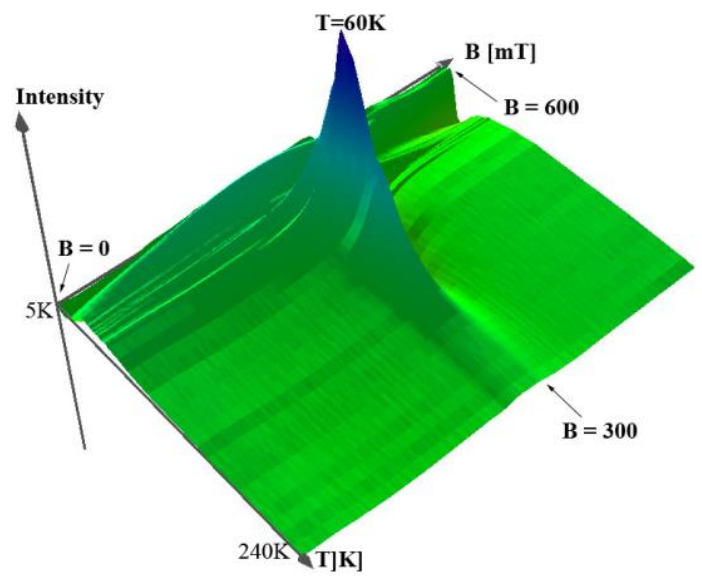

al., 2009). Here we are presenting only the main results, associated with resonance Raman data. Also some new visualizations of the EPR spectra were presented here. The EPR technique allows on probing of the active species including their local environment and dispersion in the host matrix.

Depending on the synthesis route, the EPR spectra show an exchange narrowing in one samples batch or drastic broadening and low magnetic field shift of the resonance position for others. The authors conjectured that in the samples coexist isolated $\mathrm{Ni}$-cyclam groups and Ni-cyclam clusters, where magnetic interactions hold and even show cooperative magnetic phenomena in low temperature. The proportion between isolated active molecules and clusters depending on the synthesis procedure. The EPR spectra of two batches of samples were presented in domain of magnetic field and temperature at fig. 6 and fig. 7. Presentation of EPR results as a surface caused that each characteristic phenomenon, occurred in full range of magnetic field and temperature are clearly seen.

b)

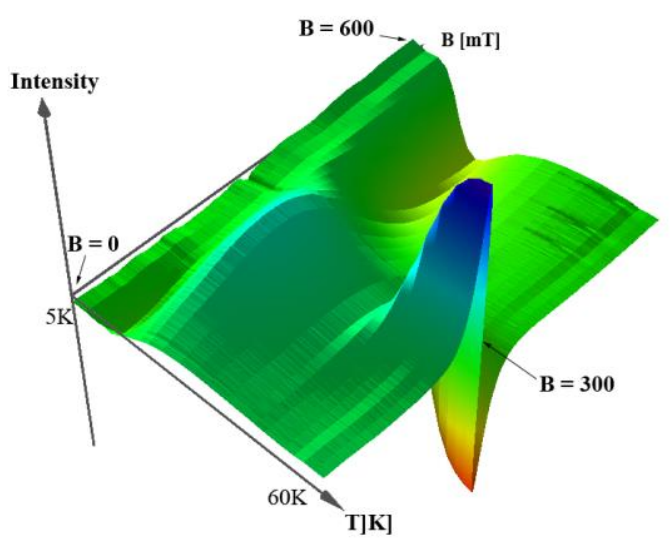

Fig. 6. Thermal evolution of EPR spectra of mesoporous silica activated by Ni-cyclam molecules (ratio 5\%) inside channels, synthesis procedure SA. EPR spectra are presented both in magnetic field and temperature domain. Full range of temperature $(a)$ and low temperature part $(b)$ of the spectrum.

In the case of SA samples, a broad EPR line can be clearly seen below $200 \mathrm{~K}$ and its intensity increases when the temperature is varied from 200 to $60 \mathrm{~K}$. Additionally, a net narrowing of the line width is observed down to $60 \mathrm{~K}$. At lower temperatures, the intensity loss, and the development of broad features indicates of magnetic ordering. The absence of anisotropy, hyperfine or superhyperfine couplings with nearest ligands such as chlorine or nitrogen ions, suggests the formation of Ni-cyclam clusters in samples. In the SA samples, the EPR spectra seem to mark spinspin exchange interactions between $\mathrm{Ni}^{3+}$.
Differently from SA samples, the SB samples shown completely different EPR features. The broad resonance features can be observed at low magnetic field. The origin of this is an agglomerated species maintaining own magnetization (see: fig. 7a). The central part of the spectra (clearly seen at fig. 7b) consists well resolved structure. The EPR signal features correlate with superhyperfine couplings with the chlorine ions located in the vicinity of $\mathrm{NI}^{3+}$ ions (confirmed by simulations Laskowski et al., 2009). 
a)

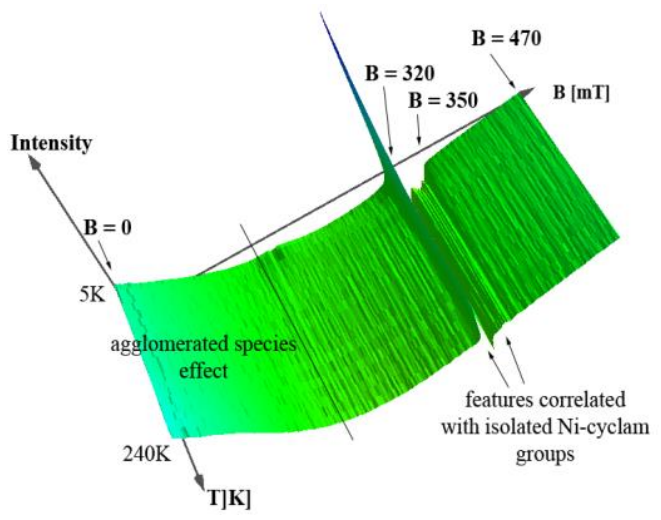

b)

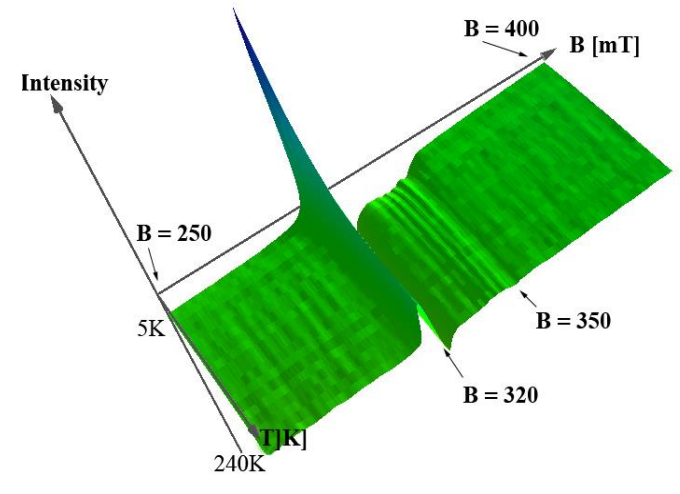

Fig. 7. Thermal evolution of EPR spectra of mesoporous silica activated by Ni-cyclam molecules (ratio 5\%) inside channels, synthesis procedure SB. At figure $a$ full range of magnetic field was presented, while figure $b$ presents the range of magnetic field cut to 250 - 400 $\mathrm{mT}$ for better visibility of coupling effect.

The magnetic ordering holds in SA samples due to the nickel-based molecules being arranged in clusters instead of being regularly dispersed as in the SB samples. Must be mentioned, that in addition to isolated Ni-cyclam centers, agglomeration of nickel-based groups are also present in SB5 sample, as identified by low magnetic field EPR signal.

\section{CONCLUSIONS}

Mesoporous silica functionalized by the Ni-cyclam groups were successfully synthesized and investigated by the resonance Raman technique. The vibrational properties of the Ni-cyclam groups in pure form and incorporated into mesoporous silica have been analyzed and interpreted. The comparative study of the two batches of samples (prepared according two different synthesis method: grafting - SA procedure or one step synthesis - SB procedure) have been carried out. Each bath of samples contained species doped by 5\% and $10 \%$ of active cyclam molecules. Results of Raman spectroscopy were compared to EPR spectroscopy findings in order to confirm the thesis about structural differences between the samples obtained by two considered here synthesis procedures.

The significant differences in Raman spectra were obtained. The Raman spectrum of SB samples shown the resonance Raman spectra containing bands which correlate with the quantity of the active groups, contrary to SA samples. In the case of SA samples the Raman spectra were similar, irrespective of the quantity of the doped compound. Authors came to a conclusion that this effect is taking from agglomeration of doping agent in the silica pores, what cannot be observed in the case of SB samples. From this study one can concluded about superiority of direct synthesis method of active mesoporous silica (SB procedure) over the metode of grafting (SA procedure).

This results are in good agreement with EPR outcomes. The EPR data clarified the effective location of nickel in the cyclam groups, their dispersion in the host silica matrices for the synthesis batch SB and the ferromagnetic exchange interactions between nearest spins in the SA batch. Indeed, in samples SA, magnetic interactions are involved and induce, at the level of clusters, a ferromagnetic ordering at low temperature. In the SB samples mainly isolated molecules of cyclam-Ni gave contribution to EPR spectrum.

Must be mentioned, that earlier UV-vis study (Laskowski, Kassiba, Makowska-Janusik, Errien, Mehdi, Swiatek, 2011) seems to confirm our dissertation.

Summing, must be stated that Raman technique can be used to probing of functionalization's efficiency of mesoporous silica, as a complementary research with EPR and other spectroscopic methods.

\section{REFERENCES}

Beck J. S., Vartuli J. C., Roth W. J., Leonowicz M. E., Kresge C. T., Schmitt K. D., C. Chu T-W., Olson D. H., Sheppard E. W., McCullen S. B., Higgins J. B., Schlenkert J. L. (1992). A New Family of Mesoporous Molecular Sieves Prepared with Liquid Crystal Templates. J.Am. Chem. Soc., 114, 10834-10843.

Zhao D., Huo Q., Feng J., Chmelka B. F., Stuc G. D. (1998). Nonionic Triblock and Star Diblock Copolymer and Oligomeric Surfactant Syntheses of Highly Ordered, Hydrothermally Stable, Mesoporous Silica Structures, J. Am. Chem. Soc.,120, 6024-6036. 
Corriu R., Mehdi A., Reye C. (2004). Nanoporous materials: a good opportunity for nanosciences. Journal of Organometallic Chemistry, 689, 4437-4450.

Corriu R. (2003a). Organosilicon chemistry and nanoscience. Journal of Organometallic Chemistry, 686, 32-41.

Laskowski L., Kassiba A., Makowska-Janusik M., Mehdi A., Gibaud A., Errien N., Swiatek J. (2009). Magnetic behaviour of nickel-cyclam complexes in mesoporous silica: EPR Investigations. J. Phys.: Condens. Matter, 21, Article Number: 076004 (9pp).

Katiyar A., Yadav S., Smirniotis G. P., Pinto N. G. (2006). Synthesis of ordered large pore SBA-15 spherical particles for adsorption of biomolecules, Journal of Chromatography, 1122, 13-20.

Trewyn B. G., Nieweg J. A., Zhao Y., Lin V. (2007). Biocompatible mesoporous silica nanoparticles with different morphologies for animal cell membrane penetration. Chemical Engineering Journal, 137, 23-29.

Slowing I. I., Trewyn B. G., Giri S., Lin V. S.-Y. (2007). Mesoporous silica nanoparticle based controlled release, drug delivery, and biosensor systems, Advanced Functional Materials, 17, 1225 - 1236.

Jung D., Hartmann M. (2008). Oxidation of Indole with CPO and GOx Immobilized on SBA-15. Studies in Surface Science and Catalysis, 174, 1045-1050

Xiao F.-S. (2005). Ordered Mesoporous Materials with Improved Stability and Catalytic Activity. Topics in Catalysis, 35, 9-24.

Lebeau B., Sanchez C. (1999). Sol-gel derived hybrid inorganic-organic nanocomposites for optics. Current Opinion in Solid State and Materials Science, 4, 11-23.

Singh V. B., Singh A. K., Amareshwar K., Rai A. K., Singh A. N., Rai D. K. (2007). Spectrochimica Acta Part A, 67, 687693.
Otakar F., Jehlicka J., Howell G.M.E. (2007). Raman spectroscopy as tool for the characterization of thiopolyaromatic hydrocarbons in organic minerals. Spectrochimica Acta Part A, 68, 1065-1069.

Xu J., Butler I. S., Gibson D. F. R., Stangel I. (1997). Highpressure infrared and FT-Raman investigation of a dental composite. Biomaterials, 18, 1653-1657.

Smierciak R., Passariello J., Blinn E.L. (1977). A comparative study of steric effects of nickel(II) complexes containing 12membered macrocyclic ligands, Inorganic Chemistry 16, 2646-2648.

Hess C., Wild U., Schlögl R. (2006). The mechanism for the controlled synthesis of highly dispersed vanadia supported on silica SBA-15, Microporous and Mesoporous Materials, 95, 339-349.

Hess C. (2006). Characterization of the synthesis and reactivity behavior of nanostructured vanadia model catalysts using XPS and vibrational spectroscopy. Surface Science, 600, 3695-3701.

Davidson G. (2005). Spectroscopic Properties of Inorganic and Organometallic Compounds. Royal Society of Chemistry, 37, 114-172.

Liao Q., Li M.Y., Hao R., Ai X.C., Zhang J.P., Wang Y. (2007). Surface-enhanced Raman scattering and DFT computational studies of a cyanuric chloride derivative. Vibr. Spectr. 44, 351-356.

Jones R.M., Goldcamp M. J., Krause J. A., Baldwin M. J. (2006). Theoretical, structural, and spectroscopic studies of a series of $\mathrm{Ni}^{\mathrm{II}}\left(\mathrm{TRISOXH}_{3}\right) \mathrm{X}_{2}$ complexes. Polyhedron 25, 3145-3158.

Laskowski L., Kassiba A., Makowska-Janusik M., Errien N., Mehdi A., Swiatek J. (2011). Synthesis and Optical Behavior of Mesoporous Silica Functionalized by Organometallic Molecules. Journal of Physics: ConferenceSeries, 289, 012024. 